Pacific Journal of Mathematics

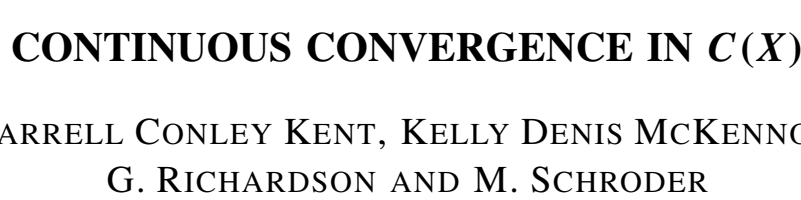




\section{CONTINUOUS CONVERGENCE IN $C(X)$}

\section{Kent, K. McKennon, G. Richardson, And M. Schroder}

Let $X$ be a convergence space and $C(X)$ the $R$-algebra of all continuous real-valued functions on $X$, equipped with the continuous convergence structure. If the natural map from $X$ into $C(C(X))$ is an embedding, then $X$ is said to be a $c$-space. With each space $X$ there is associated the $c$-modification $c X$ which is a $c$-space with the property $C(X)=C(c X)$. This leads to the following theorems which are valid for any convergence space $X:(1) C(X)$ is a topological space iff $c X$ is locally compact; $(2) C(X)$ is locally compact iff $c X$ is finite.

1. Preliminaries. The continuous convergence structure on the function algebra $C(X)$ of continuous real-valued functions on a space $X$ has been studied extensively by Binz and others during the past decade; see, for instance, [3], [4], [6], and [8]. This function space is typically called $C_{c}(X)$, but we will use instead the notation $C(X)$, assuming this space to be equipped with continuous convergence unless otherwise indicated. The term "space" will always mean "convergence space".

Let Hom $C(X)$ be the subspace of $C(C(X))$ consisting of all nonzero continuous homomorphisms on $C(X) . \quad X$ is c-embedded if the function $i_{X}: X \rightarrow \operatorname{Hom} C(X)$ (defined by $i_{X}(x)(f)=f(x)$ for all $f$ in $C(X))$ is a homeomorphism. We will use the term c-space in place of "c-embedded space"; this terminology is not only more concise, but avoids conflict with the different usage of the term "c-embedded" which is common in the literature.

Starting with a space $X$, let $\lambda X$ denote the topological modification and $\pi X$ the pretopological modification of $X$. The symbol $\omega X$ will denote the completely regular modification of $X$, that is, the finest completely regular topological space on the same underlying set which is coarser than $X . X$ will be called $\omega$-Hausdorff if $\omega X$ is Hausdorff, and $\omega$-regular if $\mathrm{cl}_{\omega X} \mathscr{F} \rightarrow x$ whenever $\mathscr{F} \rightarrow x$. (Here, " $\mathscr{F} \rightarrow x$ in $X$ " means "the filter $\mathscr{F}$ converges to $x$ in the space $X$ "; $\mathrm{cl}_{X}$ designates the closure operator for $X$.) The following proposition will be useful later in the paper.

Proposition 1.1. A Hausdorff $\omega$-regular space $X$ is $\omega$-Hausdorff.

Proof. Let $\mathscr{F}$ converge to $x$ and $y$ in $\omega X$. Since $\dot{y} \geqq \mathrm{cl}_{\omega X} \mathscr{F}$, $\dot{y} \rightarrow x$ in $\omega X$, and so $\dot{x} \geqq \operatorname{cl}_{\omega X} \dot{y}$. But $\dot{y} \rightarrow y$ in $X$, and, since $X$ is $\omega$-regular, $\dot{x} \rightarrow y$ in $X$. Therefore, $x=y$. 
A space is said to be pseudo-topological if $\mathscr{F} \rightarrow x$ whenever each ultrafilter finer than $\mathscr{F}$ converges to $x$. $X$ is locally compact if every convergent filter contains a compact set, and first countable if every convergent filter contains a filter converging to the same point which has a countable filter base. The notation " $X \leqq Y$ " for spaces $X$ and $Y$ will mean that $X$ and $Y$ have the same underlying set, and $\mathscr{F} \rightarrow x$ in $Y$ implies $\mathscr{F} \rightarrow x$ in $X$.

A collection $\mathscr{A}$ of subsets of a space $X$ is called a covering system if each convergent filter contains a member of $\mathscr{A}$. If $\mathscr{A}$ and $\mathscr{A}_{1}$ are covering systems such that each member of $\mathscr{A}_{1}$ is a subset of a member of $\mathscr{A}$, then $\mathscr{A}_{1}$ is said to be a refinement of $\mathscr{A}$.

Proposition 1.2. A subset $A$ of a space $X$ is compact iff, for each covering system $\mathscr{A}$ of $A$, there is a refinement $\mathscr{A}_{1}$ of $\mathscr{A}$ such that a finite subcollection of $\mathscr{A}_{1}$ covers $A$.

A covering system $\mathscr{B}$ for $X$ will be called a basic covering system if, whenever $\mathscr{F} \rightarrow x$, there is a filter $\mathscr{G} \rightarrow x$ such that $\mathscr{G} \leqq$ $\mathscr{F}$ and $\mathscr{G}$ has a filter base consisting of members of $\mathscr{B}$. Thus, if $X$ is $\omega$-regular, the set of all $\omega X$-closed subsets of $X$ forms a basic covering system for $X$.

The abbreviation "u.f." will be used for "ultrafilter". The symbol $R$ will denote the real number system with its usual topology. The complement of a set $A$ is written "Co $A$ ", and the symbol $\dot{x}$ represents the fixed ultrafilter generated by $\{x\}$.

2. $c$-spaces. It is shown in [8] that $X$ is a $c$-space iff $X$ is Hausdorff, $\omega$-regular, and solid (see [8] for the definition of a solid space). We will begin by showing that $X$ is a $c$-space iff $X$ is Hausdorff, $\omega$-regular, and pseudo-topological.

Proposition 2.1. A c-space is Hausdorff, $\omega$-regular, and pseudotopological.

Proof. A c-space must be Hausdorff in order for the natural function from $X$ into $\operatorname{Hom} C(X)$ to be injective. That a $c$-space is $\omega$-regular is established in [6].

To show that $X$ must be pseudo-topological, it is sufficient to show that $C(X)$ is pseudo-topological, since this property is hereditary. Let $\Lambda$ be a filter on $C(X)$, and assume $\Omega \rightarrow f$ for all u.f.'s $\Omega \geqq \Lambda$. Let $\mathscr{F} \rightarrow x$ in $X$. To show $\Lambda \rightarrow f$, it is enough to show that $\Lambda(\mathscr{F}) \geqq \bigcap\{\Omega(\mathscr{F}): \Omega$ an u.f., $\Omega \geqq \Lambda\}$. We will do this by showing that if $\mathscr{A}$ is an u.f. on $R$ and $\mathscr{A} \geqq \Lambda(\mathscr{F})$, then $\mathscr{A} \geqq \Omega(\mathscr{F})$ for some u.f. $\Omega \geqq \Lambda$. 
Let $\mathscr{Z}=\{\Sigma: \Sigma$ a filter on $C(X), \Sigma \geqq \Lambda$, and $\mathscr{A} \geqq \Sigma(\mathscr{F})\} . \quad \mathrm{A}$ standard Zorn's lemma argument establishes that contains a maximal element; call it $\Omega$.

To show that $\Omega$ is an u.f., assume that $A \cup B \in \Omega$. If neither $A$ nor $B$ is in $\Omega$, then the filter $\Omega_{A}$ generated by $\{A \cap M: M \in \Omega\}$, and $\Omega_{B}$ generated by $\{B \cap M: M \in \Omega\}$ must both be proper filters on $C(X)$ which are strictly finer than $\Omega$. Thus $\Omega_{A}$ and $\Omega_{B}$ must both fail to be in $\mathscr{Z}$, and so there are sets $M_{1}, M_{2}$ in $\Omega$ and $F_{1}$ and $F_{2}$ in $\mathscr{F}$ such that $\left(M_{1} \cap A\right)\left(F_{1}\right) \notin \mathscr{A}$ and $\left(M_{2} \cap A\right)\left(F_{2}\right) \notin \mathscr{A}$. Let $M=$ $M_{1} \cap M_{2}$ and $F=F_{1} \cap F_{2}$. But $A \cup B \in \Omega$, and so $(M \cap(A \cup B))(F) \in \mathscr{A}$. However, $(M \cap(A \cup B))(F) \subseteq\left(M_{1} \cap A\right)\left(F_{1}\right) \cup\left(M_{2} \cap B\right)\left(F_{2}\right)$, and this contradicts the fact that $\mathscr{A}$ is an ultrafilter. Thus either $A$ or $B$ must be in $\Omega$.

For any $\omega$-Hausdorff space $X$, define $\tilde{X}$ to be the space on the same underlying set with convergence defined as follows: $\mathscr{F} \rightarrow x$ in $\tilde{X}$ iff, whenever $\Lambda \rightarrow f$ in $C(X), \Lambda(\mathscr{F}) \rightarrow f(x)$ in $R$.

Proposition 2.2. The following statements are valid for any $\omega$-Hausdorff space $X$ : (a) $\omega X \leqq \widetilde{X} \leqq X$; (b) $C(\widetilde{X})=C(X)$; (c) $\widetilde{X}$ is the finest c-space coarser than $X$.

Proof. Assertions (a) and (b) are obvious. It is also clear from the definition that $\tilde{X}$ is $c$-embedded. If $X_{1}$ is $c$-embedded and $X_{1} \leqq$ $X$, then the identity map from $X \rightarrow X_{1}$ is continuous. Thus the induced $\operatorname{map} C\left(X_{1}\right) \rightarrow C(X)=C(\widetilde{X})$ is continuous, and so is the induced map from $\operatorname{Hom} C(\widetilde{X}) \rightarrow \operatorname{Hom} C\left(X_{1}\right)$. But $\widetilde{X}$ is homeomorphic to Hom $C(\widetilde{X})$ and Hom $C\left(X_{1}\right)$ is homeomorphic to $X_{1}$, which establishes $X_{1} \leqq \widetilde{X}$.

Lemma 2.3. Let $\mathscr{B}$ be a basic covering system for $X$, and let $t$ be the topology on $C(X)$ with subbase $\{(B, W): B \in \mathscr{B}, W$ open in $R\}$, where $(B, W)=\{g \in C(X): g(B) \subseteq W\}$. Then $C(X) \leqq C_{t}(X)$.

Proof. Let $\Phi \rightarrow h$ in $C_{t}(X)$ and $\mathscr{G} \rightarrow y$ in $X$; let $W$ be an open neighborhood of $h(y)$ in $R$. Let $\mathscr{H} \rightarrow y$ in $X$ such that $\mathscr{G} \geqq \mathscr{H}$, and $\mathscr{H}$ has a filter base in $\mathscr{B}$. Let $B \in \mathscr{B}$ be a basic set in $\mathscr{H}$ such that $h(B) \subseteq W$. Then $(B, W)$ is $t$-open, and hence $(B, W) \in \Phi$. But $(B, W)(B) \subseteq W$, and it follows that $\Phi(\mathscr{C}) \rightarrow h(y)$ in $R$. Thus $\Phi(\mathscr{G}) \rightarrow h(y)$ in $R$, and so $\Phi \rightarrow h$ in $C(X)$.

THEOREM 2.4. $X$ is a c-space iff $X$ is Hausdorff, $\omega$-regular, and pseudo-topological. 
Proof. Assume the three conditions. By Proposition 1.1, $X$ is $\omega$-Hausdorff. Thus $\widetilde{X}$ exists, and it is sufficient to show that $X=\widetilde{X}$. Since $X$ and $\widetilde{X}$ are both pseudo-topological, and $\widetilde{X} \leqq X$, we can complete the proof by showing that each u.f. which $\widetilde{X}$-converges to $x$ also $X$-converges to $x$.

Suppose $\mathscr{G}$ is an u.f., $\mathscr{G} \rightarrow x$ in $\tilde{X}$, and $\mathscr{G} \rightarrow x$ in $X$. If $\mathscr{H}$ is an $X$-convergent filter, then $\mathscr{G} \supsetneqq \mathrm{cl}_{\omega X} \mathscr{H}$, and so there is an $\omega X$ closed set $H$ in $\mathscr{X}$ such that Co $H \in \mathscr{G}$. The set of all such $H$ 's forms a covering system $\mathscr{A}$ for $X$, and the set $\mathscr{B}$ of all $\omega X$-closed subsets of members of $\mathscr{A}$ is a basic covering system for $X$ consisting entirely of sets whose complements are in $\mathscr{G}$. Let $C_{t}(X)$ be the topological space derived from $\mathscr{B}$ as in Lemma 2.3. Then, by the same lemma and Proposition 2.2, $C_{t}(X) \geqq C(X)=C(\tilde{X})$.

Let $f^{\prime}$ in $C(X)$ be defined by $f(x)=0$, all $x$ in $X$. Let $\Lambda$ be the $t$-neighborhood filter at $f$. Then $\Lambda \rightarrow f$ in $C_{t}(X)$, which implies $\Lambda \rightarrow$ $f$ in $C(\tilde{X})$, and so $\Lambda(\mathscr{G}) \rightarrow 0$ in $R$. Let $W$ be a neighborhood of 0 not containing 1 . Then there are sets $G$ in $\mathscr{G}$ and $L$ in $\Lambda$ such that $L(G) \cong W . L$ contains a set of the form $\left(A_{1}, V_{1}\right) \cap \cdots \cap\left(A_{n}, V_{n}\right)$, where each $A_{\imath}$ is in $\mathscr{B}$ and each $V_{\imath}$ is an open neighborhood of 0 in $R$. Since Co $A_{i} \in \mathscr{G}$ for $i=1, \cdots, n$, we can choose $G_{1} \subseteq G$ such that $G_{1}$ is in $\mathscr{G}$ and $G_{1} \cap\left(\bigcup A_{2}\right)$ is the empty set. Let $z$ be any element of $G_{1}$, and let $g$ in $C(X)$ be constructed such that $g(z)=1$ and $g\left(\cup A_{i}\right)=0$. Then $g$ is in $\left(A_{i}, V_{i}\right)$ for all $i$, but $g(G)$ is not a subset of $W$. This contradiction establishes that $\mathscr{G} \rightarrow x$ in $X$, and the proof is complete.

For any space $X$, let $c X=\operatorname{Hom} C(X)$. Then $c X$ is a $c$-space, and we will refer to it as the c-modification of $X$. The next result can be easily verified.

Proposition 2.5. (a) $C(X)$ and $C(c X)$ are homeomorphic. (b) If $X$ is $\omega$-Hausdorff, then $\widetilde{X}$ and $c X$ are homeomorphic.

When $X$ is $\omega$-Hausdorff, it is convenient to think of $c X$ as coinciding with $\tilde{X}$. In general, the underlying set for $c X$ can be thought of as consisting of equivalence classes relative to the following equivalence relation on $X: x \sim y$ iff $f(x)=f(y)$, for all $f$ in $C(X)$.

For the purpose of studying $C(X), X$ can be replaced by the $c$ space $c X$. If one wishes to determine what properties of $C(X)$ are induced by given properties of $X$, it would naturally be of interest to know when a given property of $X$ extends to $c X$. Two such properties are "Lindelof" and "second countable"; for definitions of these concepts in a convergence space setting, the reader is referred to [6]. 
Proposition 2.6. If $X$ is Lindelof (second countable), then $c X$ is Lindelof (second countable).

Proof. In Theorem 1, [6], Feldman shows that $C(X)$ is first countable whenever $X$ is Lindelof, and that $X$ is Lindelof whenever $X$ is a $c$-space and $C(X)$ is first countable. The assertion for "Lindelof" follows immediately from these results. The assertion for "second countable" can be proved similarly with the help of Theorem 2 of [6].

In the next section, we will show that $c X$ is locally compact whenever $X$ is locally compact. We conclude this section with a simple example which shows that $X$ can be first countable when $c X$ is not first countable.

ExAMPLE 2.7. Let $Y$ be the interval $[0,1]$ with the usual topo$\operatorname{logy}$. Let $X$ be the space with the same underlying set whose convergence to nonzero points is discrete, and with convergence to 0 defined as follows: $\mathscr{F} \rightarrow 0$ in $X$ iff there is a free filter $\mathscr{G}$ and a point $y$ in $Y$ such that: (1) $\mathscr{G}$ is finer than the $Y$-neighborhood filter at $y$; (2) $\mathscr{F} \geqq \mathscr{G} \cap \dot{0}$. In other words, $\mathscr{F} \rightarrow 0$ in $X$ means that $\mathscr{F}$ is finer than the $Y$-neighborhood filter at 0 , or else $\mathscr{F}$ is finer than $\mathscr{N}(y)$ for some $y$ in $X$, where $\mathscr{N}(y)$ is generated by sets of the form $(V-\{y\}) \cup\{0\}$ and $V$ is a $Y$-neighborhood of $y$.

The space $X$ is clearly compact and first countable. But $c X$ (which turns out to be finest pseudo-topological space coarser than $X)$ is homeomorphic to the one-point compactification of the interval $(0,1]$ with the discrete topology, and so is not first countable.

3. Local compactness. In this section, we examine the consequences of assuming that either $X$ or $C(X)$ is locally compact. Arens, [1], proved for a completely regular topological space $X$ that $C(X)$ is a topological space iff $X$ is locally compact. In Theorem 3.6, we show that Arens' theorem is valid in the larger class of $\omega$-regular convergence spaces. We also show that when $C(X)$ is a topology, then $C(X)$ has the compact-open topology relative to $c X$, but not, in general, relative to $X$.

Lemma 3.1. Let $X$ be a space, $\mathscr{F} \rightarrow x$ in $X$, and $\Phi \rightarrow f$ in $C(X)$. Let $W$ be an open neighborhood of $f(x)$ in $R$.

(a) If $F$ is a compact set in $\mathscr{F}$ such that $f(F) \subseteq W$, then there is a set $A$ in $\Phi$ such that $A(F) \subseteq W$.

(b) If $A$ is a compact set in $\Phi$ and $A(x) \subseteq W$, then there is a set $F$ in $\mathscr{F}$ such that $A(F) \cong W$. 
Proof. The proofs of (a) and (b) are essentially the same, so we will prove only $(b)$.

Let $\left\{\Lambda_{i}: i \in I\right\}$ be the collection of all filters on $C(X)$ which converge in $C(X)$ to a point in $A$. Let $\Lambda_{i} \rightarrow g$ in $A$; then there are sets $L_{i}$ in $\Lambda_{i}$ and $F_{i}$ in $\mathscr{F}$ such that $L_{\imath}\left(F_{\imath}\right) \cong W$. The set $\left\{L_{i}: i \in I\right\}$ is a covering system for $A$ which, by Proposition 1.2, reduces to a finite subcover $L_{1}, \cdots, L_{n}$ of $A$. Let $F_{1}, \cdots, F_{n}$ be the corresponding members of $\mathscr{F}$, and let $F=\bigcap F_{k}$. Then $\left(\bigcup L_{k}\right)(F) \subseteq W$, and so $A(F) \subseteq W$.

THEOREM 3.2. If $X$ is a locally compact space, then $C(X)$ is a topological space.

Proof. $C(X)$ is known to be a convergence group, and it is also known (see [7], Theorem 5, §3) that a pretopological convergence group is topological. Thus we can complete the proof by showing that, whenever $f$ is in $C(X), \mathscr{F} \rightarrow x$ in $X$, and $W$ is an open neighborhood of $f(x)$ in $R$, there is a neighborhood $V$ of $f$ in $C(X)$ and $F$ in $\mathscr{F}$ such that $V(F) \leqq W$. Assume that $W_{1}$ is a closed neighborhood of $f(x)$ contained in $W$ and $F_{0}$ a compact set in $\mathscr{F}$. Let $F_{1}=F_{0} \cap f^{-1}\left(W_{1}\right)$. Then $F_{1}$ is compact, $F_{1}$ is in $\mathscr{F}$, and $f\left(F_{1}\right) \subseteq W$, so that Lemma 3.1(a) can be applied to obtain, for each filter $\Lambda \rightarrow f$ in $C(X)$, a set $L_{A} \in A$ such that $L_{A}\left(F_{1}\right) \subseteq W_{1}$. If $V$ is the union of these $L_{A}$ 's, over all $\Lambda$ 's converging to $f$ in $C(X)$, then $V$ is a neighborhood of $f$, and $V\left(F_{1}\right) \subseteq W$.

The preceding proof made use of Lemma 3.1(a) to show that $C(X)=\pi C(X)$ when $X$ is locally compact. An analogous argument, based on Lemma 3.1(b), establishes the following result.

Lemma 3.3. Let $X$ be any space such that $C(X)$ is locally compact. Then $C(X)=C(\pi X)$.

Proposition 3.4. If $C(X)$ is a locally compact space, then $C(X)=$ $C(\pi X)=C(\lambda X)$.

Proof. In view of Lemma 3.3, it is sufficient to assume that $X$ is pretopological and show that $C(X)=C(\lambda X)$. Let $\Phi \rightarrow f$ in $C(X)$ and $x \in X$. Let $W$ be an open neighborhood of $f(x)$ in $R$. Select a compact set $A$ in $\Phi$ and a neighborhood $B$ of $x$ such that $A(B) \subseteq W$. Given $z$ in $B$, we can use Lemma 3.1 to find a neighborhood $B_{z}$ of $z$ such that $A\left(B_{z}\right) \subseteq W$. Let $B_{1}=\bigcup\left\{B_{z}: z\right.$ in $\left.B\right\}$; then $A\left(B_{1}\right) \subseteq W$. Next, given $z$ in $B_{1}$, use Lemma 3.1 again to find a neighborhood $C_{z}$ of $z$ such that $A\left(C_{z}\right) \subseteq W$. Let $B_{2}=\bigcup\left\{C_{z}: z\right.$ in $\left.B_{1}\right\}$; then $A\left(B_{2}\right) \subseteq W$. 
Continue in this way to obtain $B_{n}$ such that $A\left(B_{n}\right) \leqq W$ for all natural numbers $n$. Let $U=\bigcup\left\{B_{n}: n\right.$ a natural number $\}$. Then $U$ is a $\lambda X$-neighborhood of $x$ and $A(U) \subseteq W$. It follows that $\Phi \rightarrow f$ in $C(\lambda X)$, and the proof is complete.

Corollary 3.5. (a) If $X$ is locally compact, then $C(X)=\lambda C(X)$. (b) If $C(X)$ is locally compact, then $C(X)=C(\lambda X)$.

THEOREm 3.6. If $X$ is an w-regular space, then $C(X)$ is a topological space iff $X$ is locally compact.

Proof. Assume that $C(X)$ is a topological space, and let $f$ in $C(X)$ be the constant map $f(x)=0$, all $x$ in $X$. Let $W$ be any open neighborhood of 0 in $R$ not containing 1 . Let $\mathscr{F} \rightarrow x$ in $X$; since $X$ is $\omega$-regular, there is an $\omega X$-closed set $F_{0}$ in $\mathscr{F}$ such that, for some neighborhood $U_{0}$ of $f$ in $C(X), U_{0}\left(F_{0}\right) \subseteq W$. We will complete the proof by showing that $F_{0}$ is compact.

Let $\mathscr{A}$ be a covering system for $F_{0}$. Let $\mathscr{A}_{1}=\mathscr{A} \cup\left\{\right.$ Co $\left.F_{0}\right\} \cup$ $\left\{B \cup\right.$ Co $\left.F_{0}: B \in \mathscr{A}\right\}$; then $\mathscr{A}_{1}$ is a covering system for $X$. By Lemma 2 , [6], we can replace $\mathscr{A}_{1}$ by a refinement $\mathscr{A}_{2}$ composed of $\omega X$-closed sets. Let $\mathscr{B}$ be the basic covering system for $X$ obtained by adding to the collection $\mathscr{A}_{2}$ all $\omega X$-closed subsets of members of $\mathscr{X}_{2}$. If $t$ is the topology on $C(X)$ defined from $\mathscr{B}$ as in Lemma 2.3, then $C(X) \leqq C_{t}(X)$ follows from the same lemma. Thus there is a $t$-neighborhood $U_{1}$ of $f$, with $U_{1}=\bigcap\left\{\left(F_{k}, W_{k}\right): k=1, \cdots, n\right\}$, such that $U_{1} \leqq U_{0}$, where the sets $F_{1}, \cdots, F_{n}$ are $\omega X$-closed members of $\mathscr{B}$. To show that $F_{0} \subseteq \bigcup F_{k}$, assume the contrary, and let $z \in F_{0}-\left(\bigcup F_{k}\right)$. Then there is $h$ in $C(X)$ such that $h(z)=1$ and $h\left(\cup F_{k}^{\prime}\right)=0$. This yields a contradiction, since $h$ is in $U_{1}$, a subset of $U_{0}$, but $h(z)=1$ implies $U_{0}\left(F_{0}\right) \nsubseteq W$.

To conclude that $F_{0}$ is compact, let $G_{i}=F_{i} \cap F_{0}, i=1, \cdots, n$, and let $\mathscr{A}^{*}=\left\{B \cap F_{0}: B \in \mathscr{A}_{2}\right\}$. Then $\mathscr{A}^{*}$ is an $\omega X$-closed refinement of $\mathscr{A}$, and each $G_{i}$ is a subset of some member of $\mathscr{A}^{*}$. Since $F_{0} \subseteq$ $\cup G_{k}$, the compactness of $F_{0}$ follows from Proposition 1.2, and the proof is complete.

Even in the class of topological spaces, there are $\omega$-regular spaces which are not completely regular; an example of such a space can be found in [5], page 85, Ex. 4.

CoRollary 3.7. If $X$ is locally compact, then $c X$ is locally compact.

Proof. This follows because $C(X)$ is a topology (Theorem 3.2), 
$c X$ is $\omega$-regular (Proposition 2.1), and $C(c X)$ is homeomorphic to $C(X)$ (Proposition 2.5).

An immediate consequence of Theorem 2.3 and Corollary 3.7 is

Corollary 3.8. For any space $X, C(X)$ is a topological space iff $c X$ is locally compact.

$C(X)$ is said to have the compact-open topology relative to $X$ if $C_{t}(X)=C(X)$, where $C_{t}(X)$ is the topological space derived, as in Lemma 2.3, from the collection $\mathscr{B}$ of all compact subsets of $X$. When $X$ is a completely regular topological space, then it is known that $C(X)$ has the compact-open topology relative to $X$ whenever $C(X)$ is a topology. The situation for convergence spaces can be summarized as follows.

TheOrem 3.9. Let $C(X)$ be a topological space.

(a) If $X$ is $\omega$-regular, then $C(X)$ has the compact-open topology relative to $X$.

(b) $C(X)$ always has the compact-open topology relative to $c X$.

Proof. Both assertions follow from the fact that $C(X)$ has the compact-open topology relative to $X$ whenever $X$ is locally compact. Assume that $X$ is locally compact, and let $\mathscr{B}$ be the collection of all compact subsets of $X$; let $t$ be the compact-open topology on $C(X)$. $\quad C(X) \leqq C_{t}(X)$ follows from Lemma 2.3. If $\Phi \rightarrow f$ in $C(X)$ and $f \in(K, W)$, where $K \in \mathscr{B}$ and $W$ is open in $R$, then the argument used in proving Lemma 3.1 can be applied to obtain a set $A$ in $\Phi$ such that $A(K) \subseteq W$. Thus $A \subseteq(K, W)$, and $C(X)=C_{t}(X)$ is established.

It is not generally true that $C(X)$ has the compact-open topology relative to $X$ whenever $C(X)$ is topological. One can obtain a counter-example by taking $X$ to be the space of Theorem 6.21, [2].

TheOrem 3.10. $C(X)$ is locally compact iff $c X$ is finite.

Proof. If $c X$ is finite, then $C(X)=C(c X)$ is a finite dimensional topological linear space, and hence locally compact.

Conversely, assume that $C(X)$ is locally compact; for convenience, let $Y=c X$. Then $Y$ is a completely regular topological space, since $Y$ is a subspace of $C(C(X))$, which has the compact-open topology by Theorem 3.9.

Let $A=\{f \in C(X):|f(x)| \leqq 1$, for all $x$ in $X\} . A$ is evidently 
closed in $C(X)$. Note that the filter on $C(X)$ generated by $\{(1 / n) A: n=1,2, \cdots\}$ converges to the zero function; since $C(X)$ is locally compact, some set of the form $(1 / n) A$ is compact, and it follows that $A$ is compact in $C(X)$. We can also regard $A$ as a subset of the product $R^{Y} ; A$ will then be compact relative to the product topology on $R^{Y}$.

Assume that $Y$ is infinite. If $Y$ were discrete, then $C(Y)=R^{Y}$, and $C(Y)$ would not be locally compact. Thus some element $y$ in $Y$ has a neighborhood filter distinct from $\dot{y}$. For each open set $V$ in the neighborhood filter at $y$, choose $y_{V}$ in $V-\{y\}$ and $f_{V}$ in $A$ such that $f_{V}(y)=1$ and $f_{V}(Y-V)=0$. Since $A$ is compact in $R^{Y}$, the net $\left(f_{V}\right)$ has a pointwise-convergent subnet $\left(f_{V_{\alpha}}\right)_{\alpha \in \Delta}$. Let $f$ be the pointwise-convergent limit of this subnet. Then $\left(f_{V_{\alpha}}(y)\right)_{\alpha \in A} \rightarrow$ $f(y)=1$, and $\left(f_{V_{\alpha}}\left(y_{V_{\beta}}\right)\right)_{\alpha \in \Delta} \rightarrow f\left(y_{V_{\beta}}\right)=0$ for each $\beta \in \Delta$. But the net $\left(y_{\beta}\right)_{\beta \in\lrcorner} \rightarrow y$, and so $f$ is not in $C(Y)$. But $f \in A \subseteq C(Y)$, since $A$ is compact, a contradiction. It follows that $Y=c X$ is finite.

The preceding theorem and Corollary 3.8 imply that $C(X)$ is topological whenever $C(X)$ is locally compact. Combining this result with Proposition 3.4, we obtain the following.

Corollary 3.11. If $C(X)$ is locally compact, then $C(X)=$ $\lambda C(X)=C(\lambda X)$.

We would like to thank W. A. Feldman for some helpful correspondence.

\section{REFERENCES}

1. R. Arens, A topology for spaces of transformations, Annals of Math., 47 (1946), 480-495.

2. R. Arens and J. Dugundji, Topologies for function spaces, Pacific J. Math., 1 (1951), 5-31.

3. E. Binz and H. H. Keller, Funktionenräume in der Kategorie der Limesräume, Ann. Acad. Scie. Feen. A, I, 383 (1966), 1-21.

4. E. Binz, $Z u$ den Beziehungen zwischen c-einbettbaren Limesräumen und ihrer limitierten Funktionenalgebren, Math. Ann., 181 (1969), 45-52.

5. R. Engelking, Outline of General Topology, Wiley Interscience, 1968.

6. W. A. Feldman, Axioms of countability and the algebra $C(X)$, Pacific J. Math., (to appear).

7. H. R. Fischer Limesräume, Math. Ann., 137 (1959), 269-303.

8. M. Schroder, Solid convergence spaces, Bull. Australian Math. Soc., 8 (1973), 443-459.

Received September 7, 1973 and in revised form March 6, 1974.

Washington State University

East Carolina University

AND

UNIVERSITY OF WAIKATO 



\section{PACIFIC JOURNAL OF MATHEMATICS}

EDITORS

RICHARD ARens (Managing Editor)

University of California

Los Angeles, California 90024

\section{R. A. Beaumont}

University of Washington

Seattle. Washington 98105

\section{J. DugundjI}

Department of Mathematics University of Southern California Los Angeles, California 90007

D. Gilbarg and J. Milgram

Stanford University

Stanford. California 94305

\section{ASSOCIATE EDITORS}
E. F. BECKENBACH
B. H. NEUMANN
F. WOLF
K. Yoshida

\section{SUPPORTING INSTITUTIONS}

\author{
UNIVERSITY OF BRITISH COLUMBIA \\ CALIFORNIA INSTITUTE OF TECHNOLOGY \\ UNIVERSITY OF CALIFORNIA \\ MONTANA STATE UNIVERSITY \\ UNIVERSITY OF NEVADA \\ NEW MEXICO STATE UNIVERSITY \\ OREGON STATE UNIVERSITY \\ UNIVERSITY OF OREGON \\ OSAKA UNIVERSITY
}

\author{
UNIVERSITY OF SOUTHERN CALIFORNIA \\ STANFORD UNIVERSITY \\ UNIVERSITY OF TOKYO \\ UNIVERSITY OF UTAH \\ WASHINGTON STATE UNIVERSITY \\ UNIVERSITY OF WASHINGTON

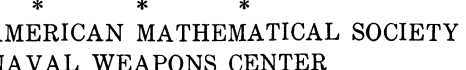

The Supporting Institutions listed above contribute to the cost of publication of this Journal, but they are not owners or publishers and have no responsibility for its content or policies.

Mathematical papers intended for publication in the Pacific Journal of Mathematics should be in typed form or offset-reproduced, (not dittoed), double spaced with large margins. Underline Greek letters in red, German in green, and script in blue. The first paragraph or two must be capable of being used separately as a synopsis of the entire paper. Items of the bibliography should not be cited there unless absolutely necessary, in which case they must be identified by author and Journal, rather than by item number. Manuscripts, in duplicate if possible, may be sent to any one of the four editors. Please classify according to the scheme of Math. Rev. Index to Vol. 39. All other communications to the editors should be addressed to the managing editor, or Elaine Barth, University of California, Los Angeles, California, 90024.

100 reprints are provided free for each article, only if page charges have been substantially paid Additional copies may be obtained at cost in multiples of 50 .

The Pacific of Journal Mathematics is issued monthly as of January 1966. Regular subscription rate: $\$ 72.00$ a year (6 Vols., 12 issues). Special rate: $\$ 36.00$ a year to individual members of supporting institutions.

Subscriptions, orders for back numbers, and changes of address should be sent to Pacific Journal of Mathematics, 103 Highland Boulevard, Berkeley, California, 94708.

\section{PUBLISHED BY PACIFIC JOURNAL OF MATHEMATICS, A NON-PROFIT CORPORATION}

Printed at Kokusai Bunken Insatsusha (International Academic Printing Co., Ltd.), 270, 3-chome Totsuka-cho. Shinjuku-ku, Tokyo 160. Japan.

Copyright (C) 1973 by Pacific Journal of Mathematics Manufactured and first issued in Japan 


\section{Pacific Journal of Mathematics}

\section{Vol. 52, No. $2 \quad$ February, 1974}

Harm Bart, Spectral properties of locally holomorphic vector-valued functions .....

J. Adrian (John) Bondy and Robert Louis Hemminger, Reconstructing infinite

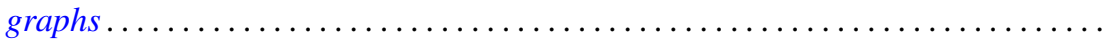

Bryan Edmund Cain and Richard J. Tondra, Biholomorphic approximation of planar

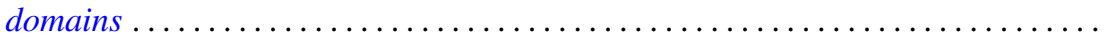

Richard Carey and Joel David Pincus, Eigenvalues of seminormal operators,

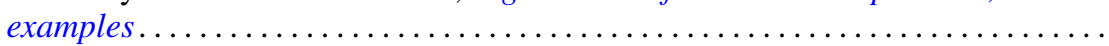

Tyrone Duncan, Absolute continuity for abstract Wiener spaces . . . . . . . . . . . . Joe Wayne Fisher and Louis Halle Rowen, An embedding of semiprime

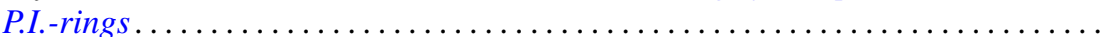

Andrew S. Geue, Precompact and collectively semi-precompact sets of semi-precompact continuous linear operators. . . . . . . . . . . . . . . .

Charles Lemuel Hagopian, Locally homeomorphic $\lambda$ connected plane continua ..... . Darald Joe Hartfiel, A study of convex sets of stochastic matrices induced by

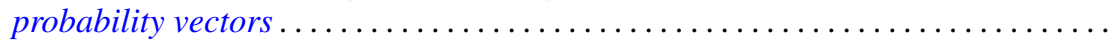

Yasunori Ishibashi, Some remarks on high order derivations $\ldots \ldots \ldots \ldots \ldots \ldots \ldots$ Donald Gordon James, Orthogonal groups of dyadic unimodular quadratic forms.

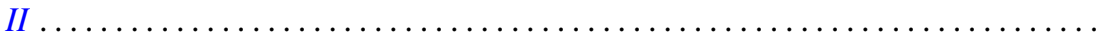

Geoffrey Thomas Jones, Projective pseudo-complemented semilattices . . . . . . . . . Darrell Conley Kent, Kelly Denis McKennon, G. Richardson and M. Schroder,

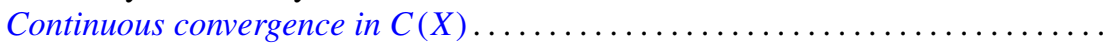

J. J. Koliha, Some convergence theorems in Banach algebras ...

Tsang Hai Kuo, Projections in the spaces of bounded linear oper

George Berry Leeman, Jr., A local estimate for typically real functions . .

475

Andrew Guy Markoe, A characterization of normal analytic spaces by the

homological codimension of the structure sheaf .........

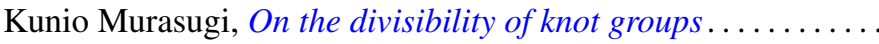

John Phillips, Perturbations of type I von Neumann algebras.

Billy E. Rhoades, Commutants of some quasi-Hausdorff matrices . .

David W. Roeder, Category theory applied to Pontryagin duality

Maxwell Alexander Rosenlicht, The nonminimality of the differential closure .

Peter Michael Rosenthal, On an inversion theorem for the general Mehler-Fock transform pair.

Alan Saleski, Stopping times for Bernoulli automorphisms

John Herman Scheuneman, Fundamental groups of compact complete locally affine complex surfaces. II. ........................

Vashishtha Narayan Singh, Reproducing kernels and operators with a cyclic vector. I. .

Peggy Strait, On the maximum and minimum of partial sums of random variables.

J. L. Brenner, Maximal ideals in the near ring of polynomials modulo 2 .

Ernst Gabor Straus, Remark on the preceding paper: "Ideals in near rings of polynomials over a field" ..........................

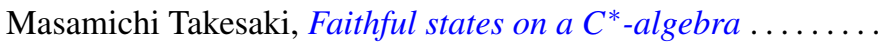

R. Michael Tanner, Some content maximizing properties of the regular simplex.

Andrew Bao-hwa Wang, An analogue of the Paley-Wiener theorem for certain

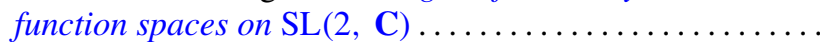

